\title{
Performance Analysis of Route Redistribution among Diverse Dynamic Routing Protocols based on OPNET Simulation
}

\author{
Zeyad Mohammad ${ }^{1}$ \\ Faculty of Science and Information Technology \\ Al Zaytoonah University of Jordan \\ Amman, 11733 Jordan \\ Ahmad Abusukhon ${ }^{2}$ \\ Faculty of Science and Information Technology \\ Al Zaytoonah University of Jordan \\ Amman, 11733 Jordan
}

\author{
Adnan A. Hnaif ${ }^{3}$ \\ Faculty of Science and Information Technology \\ Al Zaytoonah University of Jordan \\ Amman, 11733 Jordan \\ Issa S. Al-Otoum ${ }^{4}$ \\ Faculty of Science and Information Technology \\ Al Zaytoonah University of Jordan \\ Amman, 11733 Jordan
}

\begin{abstract}
Routing protocols are the fundamental block of selecting the optimal path from a source node to a destination node in internetwork. Due to emerge the large networks in business aspect thus; they operate diverse routing protocols in their infrastructure. In order to keep a large network connected; the implementation of the route redistribution is needed in network routers. This paper creates the four scenarios on the same network topology by using Optimized Network Engineering Tools Modeler (OPNET 14.5) simulator in order to analyze the performance of the route redistribution among three routing protocols by configuring three protocols from a set of Routing Information Protocol (RIPv2), Enhanced Interior Gateway Routing Protocol (EIGRP), Open Shortest Path First (OSPF), and Intermediate System to Intermediate System (IS-IS) dynamic routing protocols on each scenario. The first scenario is EIGRP_OSPF_ISIS, the second scenario is EIGRP_OSPF_RIPv2, the third scenario is RIPv2_EIGRP_ISIS, and the fourth scenario is RIPv2_OSPF_ISIS. The simulation results showed that the RIPv2_EIGRP_ISIS scenario outperforms the other scenarios in terms of network convergence time, the hops number, jitter, packet delay variation, packet end to end delay; therefore, it fits real time applications such as voice and video conferencing. In contrast, the EIGRP_OSPF_ISIS scenario has better results compared with other scenarios in terms of response time in case of using web browsing, database query, and Email services.
\end{abstract}

Keywords-route redistribution; dynamic routing protocols; EIGRP; IS-IS; OSPF; RIPv2

\section{INTRODUCTION}

Nowadays, Internet has transformed the life style from a classical environment to a technology based one. Due to the importance of routing protocols in Internet infrastructure, several routing issues and requirements must be considered in a network design phase. A Routing is a fundamental process for selecting optimal path from source to destination nodes. Routing protocols consist of interior and exterior gateway protocols. Border Gateway Protocol (BGP) is an exterior gateway protocol. BGP is designed to exchange routing information among autonomous system (AS) on the Internet. It is considered a distance vector routing protocol. An Interior Gateway Protocol is used to exchange routing information between gateways within an AS. It consists of distance vector and link state routing protocols. A distance vector algorithm builds a vector that contains costs to all other nodes and distributes a vector to its neighbors. A link state algorithm in which each node finds out the state of the link to its neighbors and the cost of each link. A distance vector routing protocol is a hop count metrics and the next hop presents a direction. It is based on Bellman Ford algorithm to calculate the optimal path. RIP is a distance vector routing protocol that measures its metrics by counting the number of hops between source and destination. It selects the minimum number of hops for reaching a destination. RIP has three versions; this study will consider RIPv2 only in a simulation. EIGRP is a distance vector routing protocol that it uses diffusion update algorithm to select the minimum cost between source and destination. A link state routing protocol is based on Dijkstra's algorithm to find a shortest path between source and destination. OSPF and IS-IS are a link state routing protocol. The enterprise networks are created from numerous routers that are running diverse routing protocols in order to exchange their route information; the configuration of the route redistribution in routers are needed. The route redistribution exchanges the route information between two different routing protocols that requires a common border router. A common border router runs routing processes in both routing protocols. The border router may be configured to redistribute routes from one routing protocol to the other, and vice versa. The route redistribution is needed in case of company mergers, multiple departments managed by multiple network administrators, multi-vendor environment, and split of two independent routing domains [1-2]. The route redistribution has two main goals. The first goal is to advertise routing information between different routing protocols for connectivity purposes. The second goal is route back up in case of a network failure, routing protocol should support alternate forwarding paths to each other. Moreover, most of existing solutions apply to 
scenarios with only two routing protocols, but large operational networks usually include more than two routing protocols [3].

The route redistribution might raise issues during running multiple different routing protocols due to each routing protocol has its characteristics such as metrics, administrative distance, convergence rate, classful and classless capabilities. Each routing protocol uses different metrics in order to calculate the optimal path. RIPv2 uses a hop count in its metric, and its administrative distance is 120, but EIGRP uses bandwidth, delay, reliability, load, and maximum transmission unit (MTU) in its metric, where bandwidth and delay are default metric in EIGRP, and its administrative distance is 90 [4]. OSPF metric is based on bandwidth, and its administrative distance is 110 , but IS-IS metric is based on cost of link utilization, delay, expense and error, where Cisco implementation uses cost only, and its administrative distance is 115 [5-6].

Each routing protocol has a different network convergence time such as EIGRP convergence time is faster than RIP. A network convergence is the status of a group of routers that have the same topological information about network in which they work. When a link fails or recovers thereafter a set of routers needs to run their routing protocols in order to exchange their routing information with neighbors to form the same topological information about their network.

Many researchers have analyzed and compared the performance of the link state and distance vector dynamic routing protocols, and the route redistribution between two diverse routing protocols [7-29]. In an enterprise network might contain more than two diverse routing protocols in order to operate. This study focuses on analyzing and comparing the performance of the route redistribution among three different routing protocols that operate in an enterprise network. This paper creates the four scenarios on the OPNET 14.5 simulator in order to analyze the performance of diverse combinations of different routing protocols that operate in the same network. The first scenario is named by EIGRP_OSPF_RIPv2 that is configured from EIGRP, OSPF and RIPv2 routing protocols in the network topology. The RIPv2_OSPF_ISIS scenario is configured from RIPv2, OSPF and IS-IS routing protocols that is a second scenario. The third scenario is named by RIPv2_EIGRP_ISIS that is configured from RIPv2, EIGRP and IS-IS routing protocols. The fourth scenario is named by EIGRP_OSPF_ISIS that is configured from EIGRP, OSPF and IS-IS routing protocols. The goal of this paper is to analyze the performance of the four scenarios in terms of convergence duration time, number of hops, voice jitter, voice and video conferencing packet delay variations, voice and video conferencing packet end to end delays, remote login, database query, HTTP object, HTTP page, Email upload and Email download response times.

The rest of the paper is organized as follows: Section 2 presents a review briefly about the performance analysis of dynamic routing protocols and the route redistribution of different routing protocols. Section 3 describes the four scenarios of the designed network topology that have been created by the OPNET 14.5 simulator. A performance analysis of the four scenarios and their results discussion are presented in section 4. The conclusion and future works are presented in section 5 .

\section{RELATED WORKS}

Abdulkadhim analyzed the performance of EIGRP, OSPF and RIP dynamic routing protocols in terms of the network convergence activity and time by using the OPNET simulator. He showed that OSPF has faster convergence time than RIP, and OSPF convergence activity is much more than RIP, therefore, OSPF can react more quickly in case of link failure [7]. Kodzo et al. simulated EIGRP, OSPF and their combination in OPNET. They analyzed the performance of EIGRP, OSPF and EIGRP_OSPF for real time application. They found that EIGRP_OSPF has less end to end delay, packet delay variation and packet loss for real application than both EIGRP and OSPF, and the combination of EIGRP and OSPF has maximum throughput than EIGRP and OSPF [8]. Mardedi and Rosidi presented the analysis and comparison of performance between EIGRP and OSPF based on Cisco Packet Tracer 6.0.1. They found that EIGRP is better than OSPF in terms of delay and convergence time [9]. Whitfield and Zhu compared the performance of OSPFv3 and EIGRPv6 by using real Cisco hardware in experiments. They noticed that EIGPRv6 outperforms OSPFv3 in terms of start-up and reconvergence speed but EIGRPv6 authentication mechanism negatively affected its performance, in contrast IP Security (IPSec) in OSPFv3 improved its performance [10]. Dey et al. presented a simulation based on Cisco Packet Tracer for dynamic routing protocols and redistribution among the protocols [11]. Patel et al analyzed the performance of OSPF and EIGRP routing protocols in terms of route summarization and route redistribution in Graphical Network Simulator (GNS3) [12]. Farhangi et al. presented the OPNET simulation based of a combination of EIGRP, OSPF and IS-IS routing protocols in a semi-mesh topology. A simulation showed that the performance of the mixed three protocols EIGRP, OSPF and IS-IS in terms of end to end delay, packet delay variation, Voice Jitter and Link throughput outperforms the other two combination of the same three routing protocols [13]. Jalali et al. evaluated the performance of RIP, OSPF, IGRP and EIGRP in terms of convergence, throughput, queuing delay, end to end delay and utilization by using the OPNET simulator. They found that EIGRP outperforms other routing protocols in their study [14]. Ashoor presented a survey in distance vector and link state dynamic routing protocols. She analyzed the performance of distance vector and link state algorithms in a mesh network [15]. Kuradusenge and Hanyuwimfura presented a comparative analysis of EIGRP configuration on IPv4 and IPv6 by modifying its metric of different values of composite metric to path selection [16]. Kaur and Mir demonstrated a comparative performance analysis of EIGRP, RIP and OSPF by using the OPNET simulator. They concluded that EIGRP is better than OSPF and RIP in terms of network convergence, throughput, utilization, queuing delay, HTTP page response and email upload response time [17]. Singh et al. configured EIGRP on IPv6 by using Cisco Packet Tracer simulator and evaluated the performance of EIGRP in IPv6 for small network [18]. Pavani et al. surveyed the performance of dynamic routing protocols in terms of router updates, link utilization and end to end delay [19]. Priyadhivya and Vanitha simulated RIP 
and OSPF in IPv6 configuration by using GNS3 emulator. They analyzed the performance of OSPF and RIP in terms of convergence and packet loss, and their result showed that OSPF has faster convergence and less packet loss [20]. Shah and Rana analyzed the convergence time of OSPF and RIP by using the OPNET. They found that OSPF single area convergence time outperforms OSPF multi area and OSPF multi stub area and the convergence time of RIP is better inside network core than outside network core [21]. Vissicchio et al. presented a study in the route redistribution with safe router configuration, and they demonstrated the self-sustained routing loops and sub-optimal routing paths problems that might occur [22]. Ud Din et al. evaluated the performance of RIP, OSPF, IGRP, and EIGRP in terms of packets dropping, traffic received, end to end delay, and jitter. They used OPNET to simulate the network in their study and they found that IGRP outperforms the other routing protocols in their simulation [23]. Kaur and Singh presented a comparative performance analysis of IS-IS, OSPFv3 and the combination of IS-IS and OSPFv3 by using the OPNET simulator. They found that IS-IS protocol is better than others in terms of video end to end delay, OSPFv3 is better in jitter and IS-IS_OSPFv3 is better in voice end to end delay[24]. ShewayeSirika and SmitaMahajine studied RIP, EIGRP and OSPF in details and simulated these routing protocols on the OPNET and Cisco Packet Tracer simulators in order to compare their performance in terms of real time applications. They concluded that RIPv2 is suitable for small network and OSPF fits large network [25]. Gehlot and Barwar compared and evaluated the performance of EIGRP and OSPF by using best effort and quality of service method in OPNET simulator. They found that EIGRP outperforms OSPF performance in both quality of service and best effort [26]. NavaneethKrishnan et al. compared EIGRP and OSPF in terms of resource usage by using Cisco Packet Tracer. They found that EIGRP uses fewer resources than OSPF [27]. Al-Hadidi et al. presented a comparative performance evaluation between OSPF and EIGRP by using the OPNET and GNS3. They concluded that the performance of EIGRP is better than OSPF in real time application [28]. Kumar et al. implemented an experiment of route redistribution between EIGRP and OSPF routing protocol in computer network using GNS3 emulator [29].

\section{THE PROPOSED NETWORK TOPOLOGY}

In order to analyze the performance of the route redistribution among three different routing protocols that is mixed from RIPv2, OSPF, EIGRP, and IS-IS. Four scenarios were created and implemented in the same network topology. The proposed network topology in this study consists of 15 routers, where R6 and R10 are the border routers that are used to exchange different routing information among the other routers, two switches, six servers, two work stations, four LAN 100BaseT local area networks, where 100BaseT_LAN object presents a fast Ethernet in a switched topology, Point to Point Digital Signal (PPP DS3) link is used to connect routers in which it supports $44.736 \mathrm{Mbps}$ data rate, Ethernet 100BaseT is used to connect other components in our simulation, where 100BaseT duplex link presents Ethernet connection with 100 Mbps speed. The six servers in the proposed network topology consists of two servers that provide multimedia services, where voice Server provides voice with pulse code modulation (PCM) Quality and Silence Suppressed, and video server supports video conferencing with high resolution video, Email, remote login and database servers provide services with high load traffic, HTTP server supports web service with heavy browsing. In order to analyze the network convergence duration time of the proposed network, a Failure Recovery node is used in the proposed network in order to simulate of fails in links of the real communication networks. The link between R6 and R8 nodes is an important communication link in the proposed network due to the path between source and destination nodes is the shortest path $(\mathrm{R} 10 \rightarrow \mathrm{R} 8 \rightarrow \mathrm{R} 6)$ as compared with the other path $(\mathrm{R} 10 \rightarrow \mathrm{R} 9 \rightarrow \mathrm{R} 7 \rightarrow \mathrm{R} 6)$, so during the simulations, we apply failure recovery events as shown in Table 1, where the time is given in second. The total simulation time for each scenario is taken to be 15 minutes.

TABLE I. LINK FAILURE AND RECOVERY BETWEEN R6 AND R8

\begin{tabular}{|l|l|}
\hline Failure & Recovery \\
\hline 120 & 300 \\
\hline 420 & 480 \\
\hline 540 & 570 \\
\hline 630 & 640 \\
\hline 700 & 705 \\
\hline 765 & 766 \\
\hline
\end{tabular}

Fig. 1 shows the EIGRP_OSPF_RIPv2 scenario that is a combination of EIGRP, OSPF, and RIPv2 routing protocols. The R6 and R10 are the border routers that are used to distribute different routing information among the other routers, where R6 is used to distribute EIGRP and OSPF, in the other side R10 distributes RIPv2 and OSPF routing information.

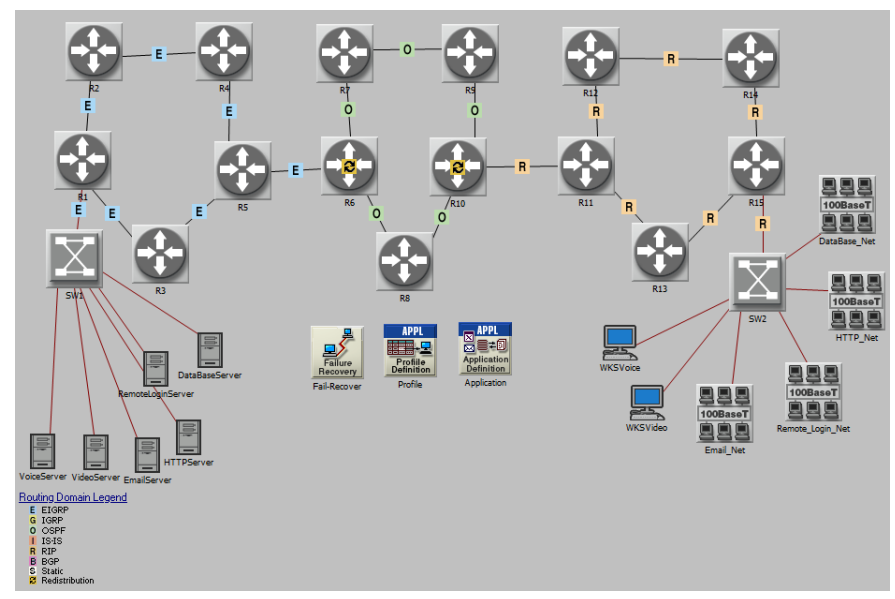

Fig. 1. The Route Redistribution among EIGRP, OSPF, and RIPv2

The RIPv2_OSPF_ISIS scenario is a combination of RIPv2, OSPF and IS-IS routing protocols that is shown in Fig. 2. In this scenario, R6 is used to advertise RIPv2 and OSPF in the proposed network, in the other side R10 is used to advertise OSPF and IS-IS routing information.

The RIPv2_EIGRP_ISIS scenario is depicted in Fig. 3, the route redistribution among RIPv2 and EIGRP is used by R6 router and R10 is used to distribute EIGRP and IS-IS routing information to the other side in the proposed network. 


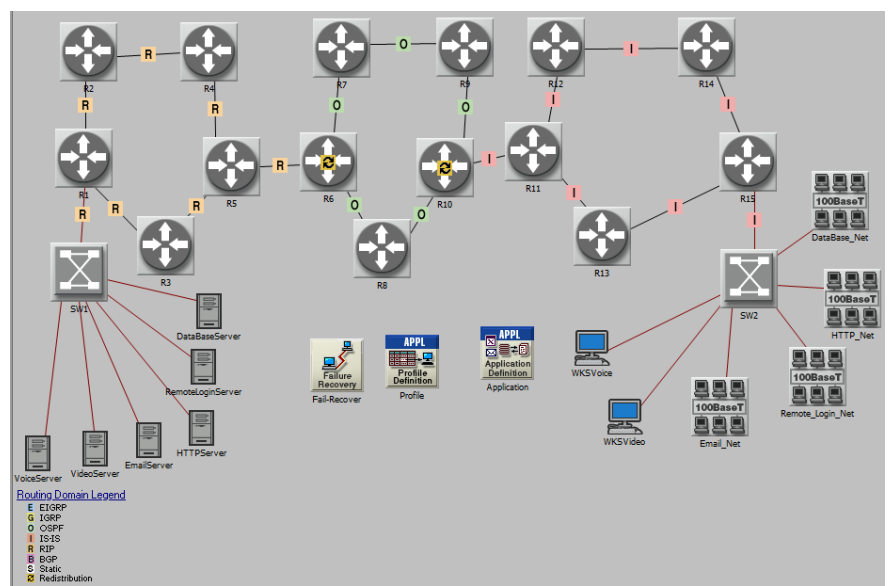

Fig. 2. The Route Redistribution among RIPv2, OSPF, and IS-IS

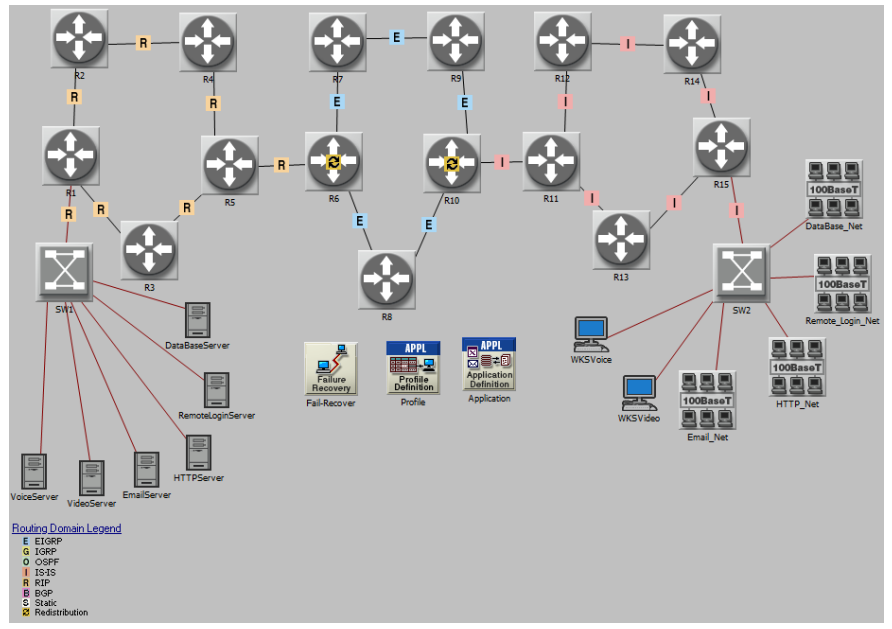

Fig. 3. The Route Redistribution among RIP, EIGRP, and IS-IS

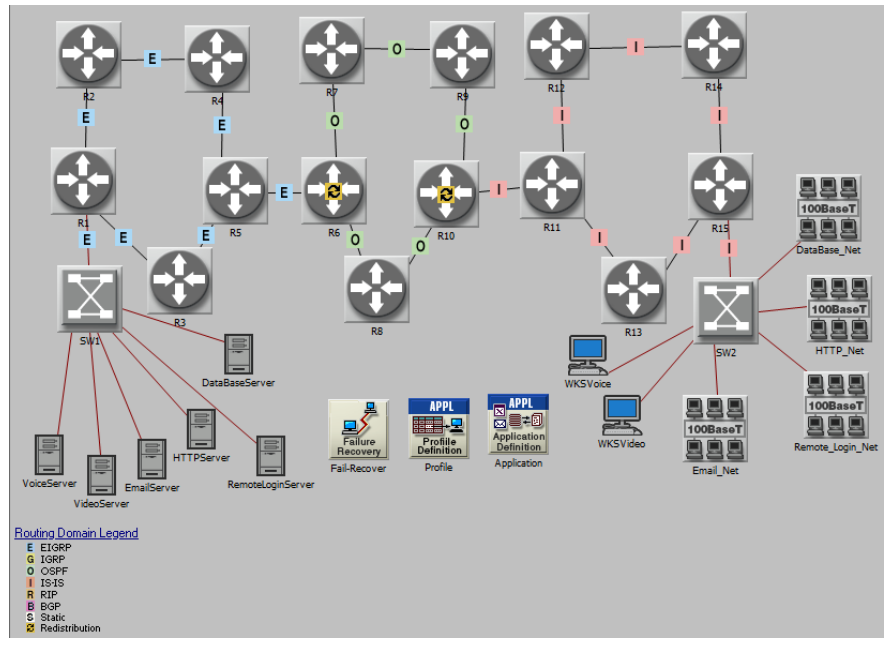

Fig. 4. The Route Redistribution among EIGRP, OSPF, and IS-IS

Fig. 4 shows the fourth scenario EIGRP_OSPF_ISIS, where the R6 is the border router that is used to advertise different routing information from EIGRP and OSPF, on the other side the R10 is the border router that is used to distribute OSPF and IS-IS routing information in the proposed network.

\section{RESULTS AND DISCUSSION}

This section presents the results that obtained from the simulations of the four scenarios in this study, therefore, the simulation results are analyzed and compared for the proposed scenarios then a decision is made about the scenarios in terms of the fitting applications for each scenario.

\section{A. Network Convergence Time}

A Failure Recovery node is applied in the proposed network as shown in Table 1 in order to analyze an average convergence duration time of the simulated network topology in this paper, where a convergence time is a measure of a time that a set of routers need to converge the network to a stable status, and a convergence duration time demonstrates how fast the convergence to reach a stable state in the network. Fig. 5 shows the RIPv2_EIGRP_ISIS scenario that has less convergence time compared with the other scenarios in the sense of the RIPv2_EIGRP_ISIS scenario has the smallest value of a convergence duration time before a failure to be occurred in the network and after network recovery among the other scenarios. Therefore, a convergence duration time in the route redistribution among three protocols RIPv2, EIGRP, and IS-IS is the fastest one in network convergence time.

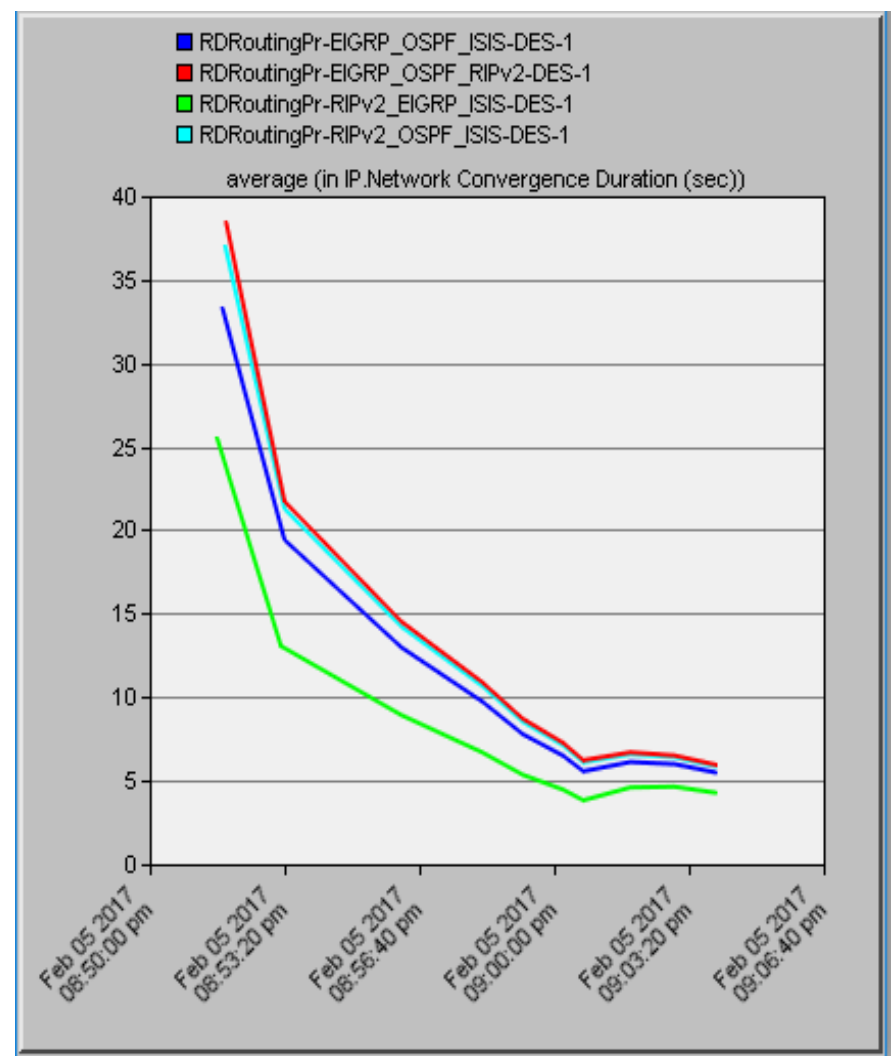

Fig. 5. An Average Convergence Duration Time in the Four Scenarios

\section{B. Hops Number}

Fig. 6 shows the RIPv2_EIGRP_ISIS scenario that has the optimal path compared with the other scenarios, because the hops number in the RIPv2_EIGRP_ISIS scenario are 10 hops before a failure to be occurred and it has the same number of hops after network recovery, but the other scenarios have 10 
hops number before a failure to be happened and they have 11 hops after network recovery. Therefore, the shortest path of the route redistribution among three protocols RIPv2, EIGRP, and IS-IS is the best one.

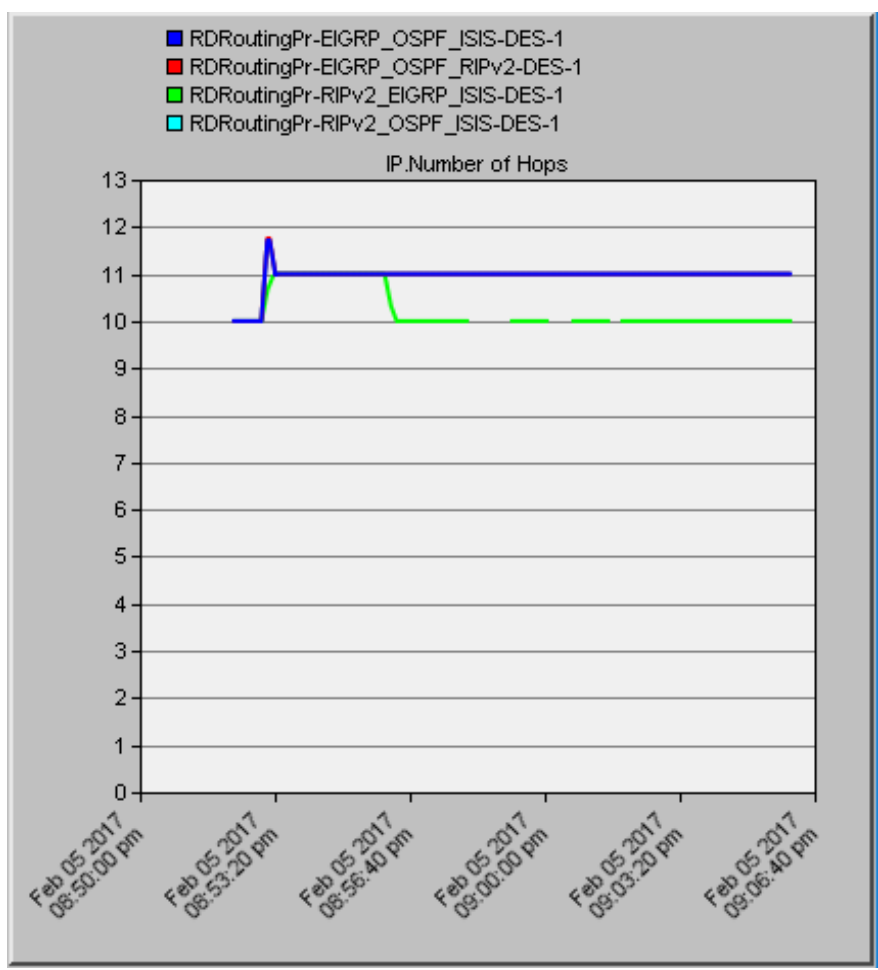

Fig. 6. The Hops Number in the Four Scenarios

\section{Response Time}

The remote login service in the RIPv2_EIGRP_ISIS scenario has the worst response time compared with the others three scenarios. In contrast, the EIGRP_OSPF_RIPv2 scenario is the fastest response time in the case of the remote login service; therefore, it is the best one as shown in Fig. 7.

Fig. 8 shows the EIGRP_OSPF_ISIS scenario that has less response time as compared with the three other scenarios, therefore, the route redistribution among three protocols EIGRP, OSPF, and IS-IS is the best in terms of using a service of data base query.

Fig. 9 shows the four scenarios in term of the HTTP object response time, where the route redistribution among three protocols RIPv2, OSPF, and IS-IS in the scenario RIPv2_OSPF_ISIS has the worst object response time among the three other scenarios. In contrast, The EIGRP_OSPF_ISIS scenario has less response time in terms of the HTTP object service, therefore, the scenario EIGRP_OSPF_ISIS is the best one.

The RIPv2_EIGRP_ISIS scenario is the slowest response time in terms of the HTTP page service as shown in Fig. 10. On the other hand, the three other scenarios have faster response time and their response time are the same; therefore, they are the best in this service.

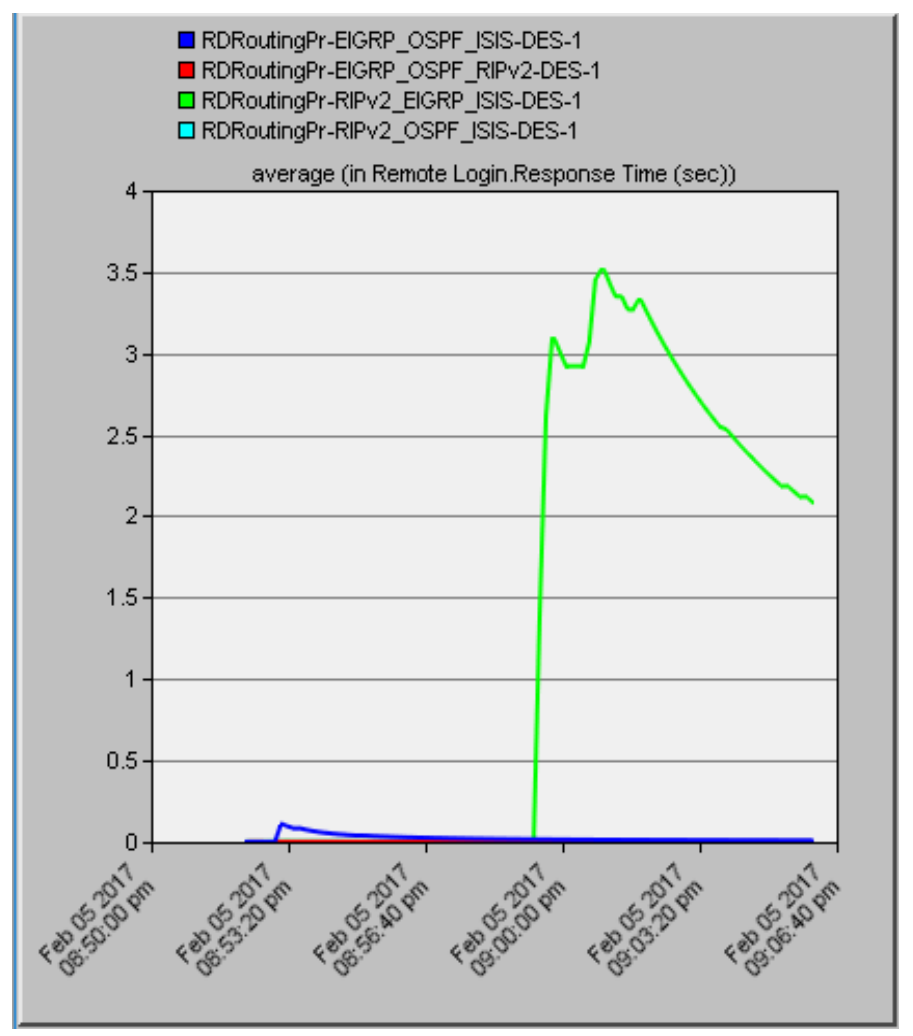

Fig. 7. The Remote Login Response Time in the four scenarios

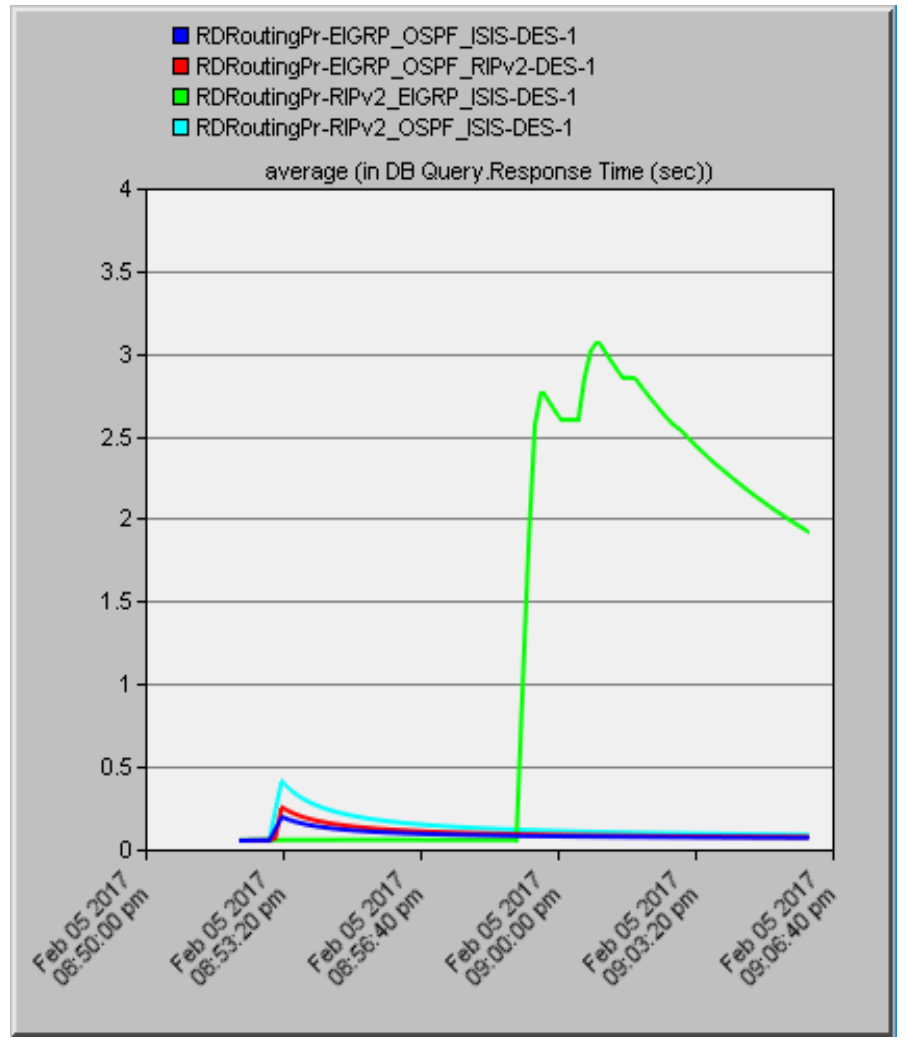

Fig. 8. The Data base Query Response Time in the four scenarios 


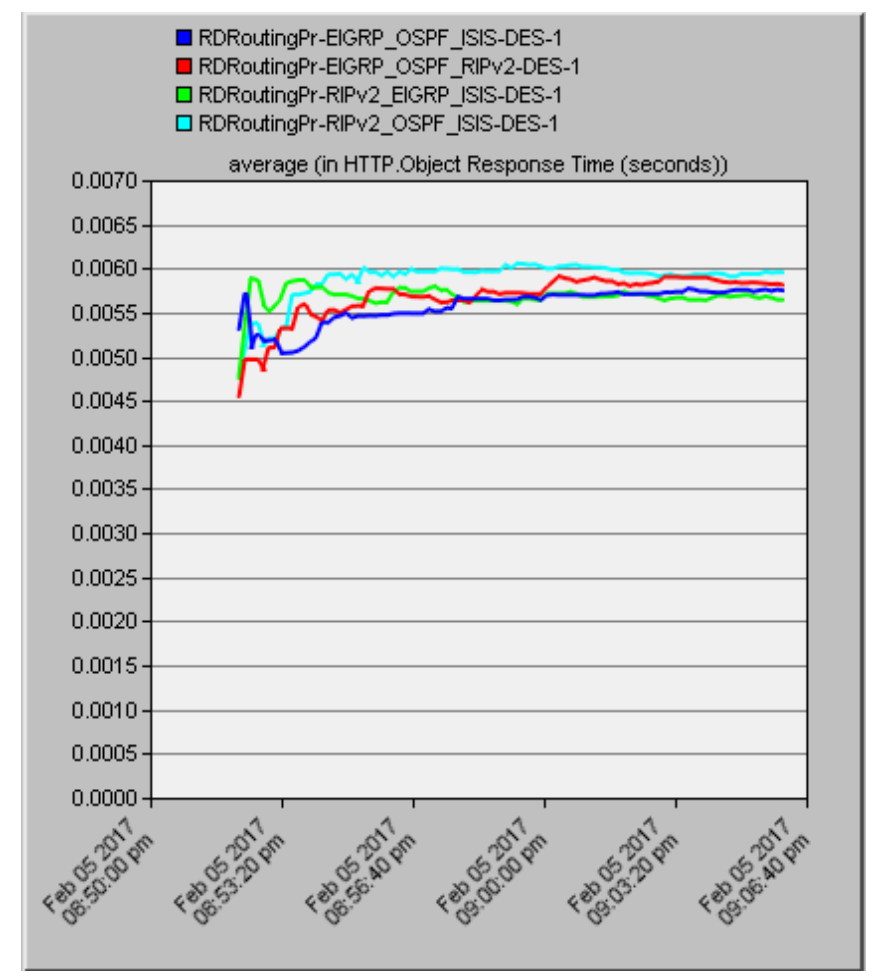

Fig. 9. The HTTP Object Response Time in the four scenarios

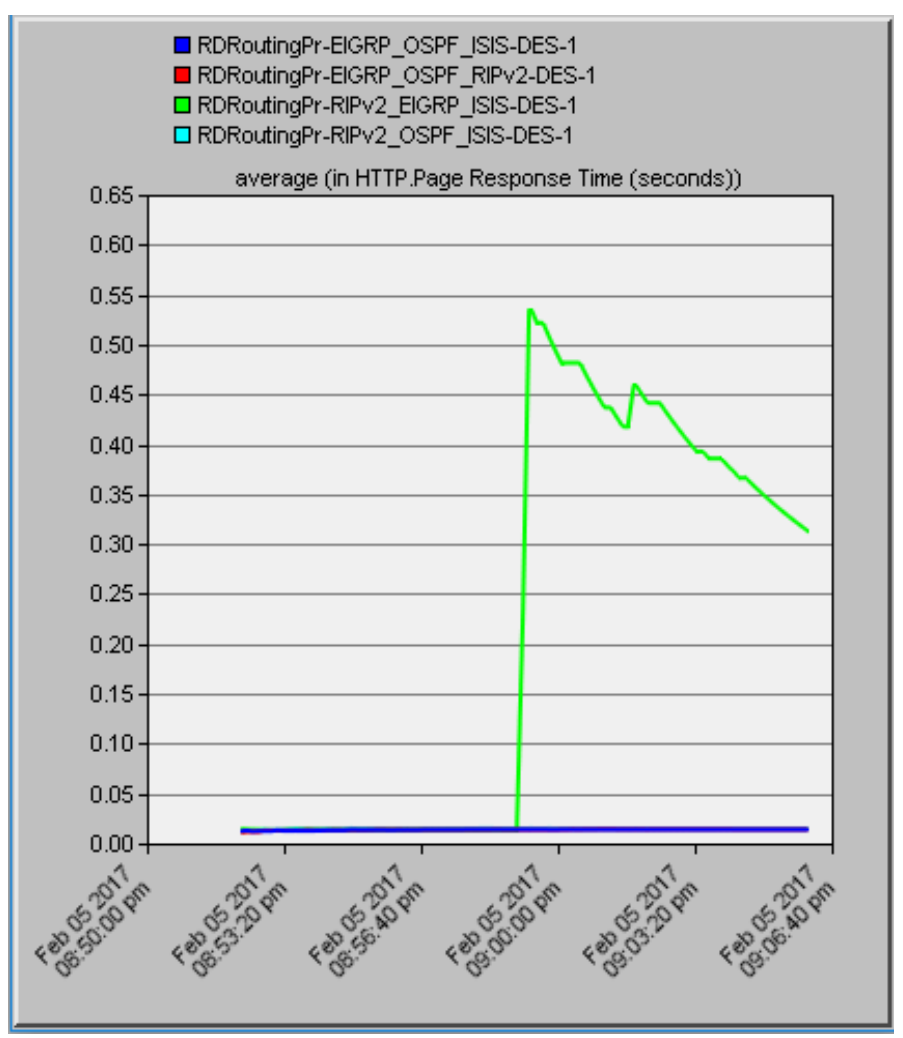

Fig. 10. The HTTP page response time in the four scenarios

Fig. 11 shows the RIPv2_EIGRP_ISIS scenario that has the worst response time compared with the three other scenarios. In contrast, the three other scenarios are faster than the RIPv2_EIGRP_ISIS scenario in terms of using the Email upload service that have the same response time thus they have the best response time.

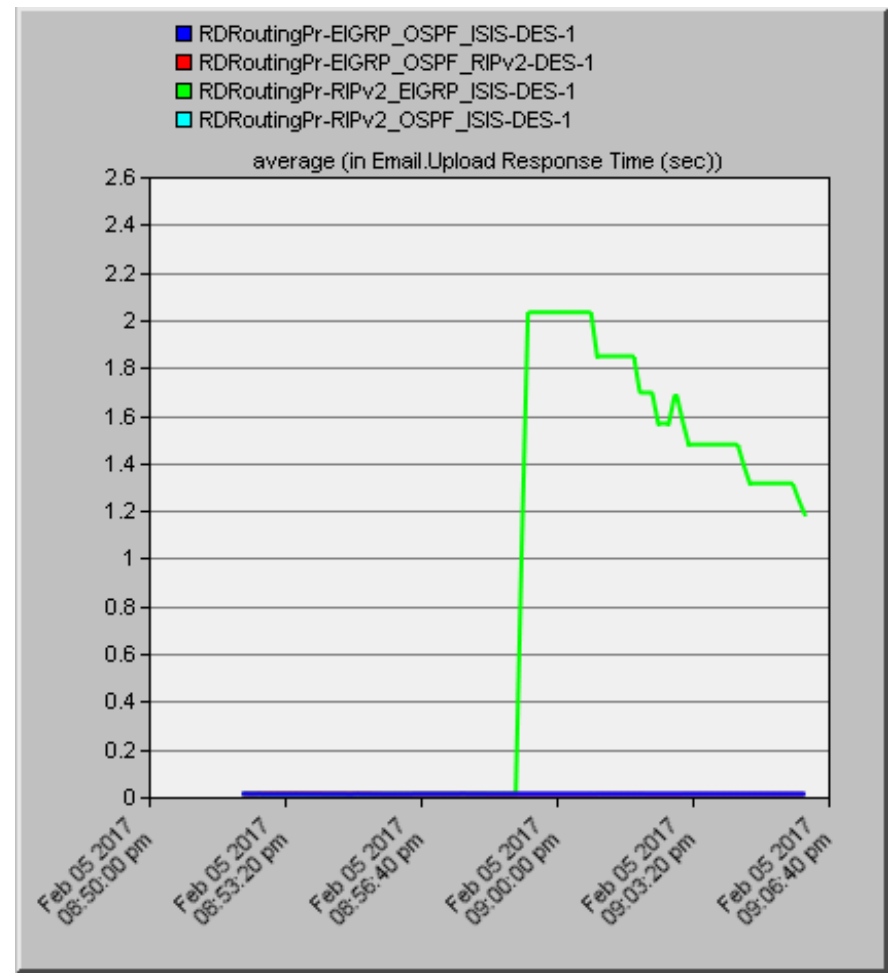

Fig. 11. The Email Upload Response Time in the four scenarios

The performance analysis in term of Email download response time is showed in Fig. 12, where the RIPv2_EIGRP_ISIS scenario has the worst result as compared with the three other scenarios in this simulation.

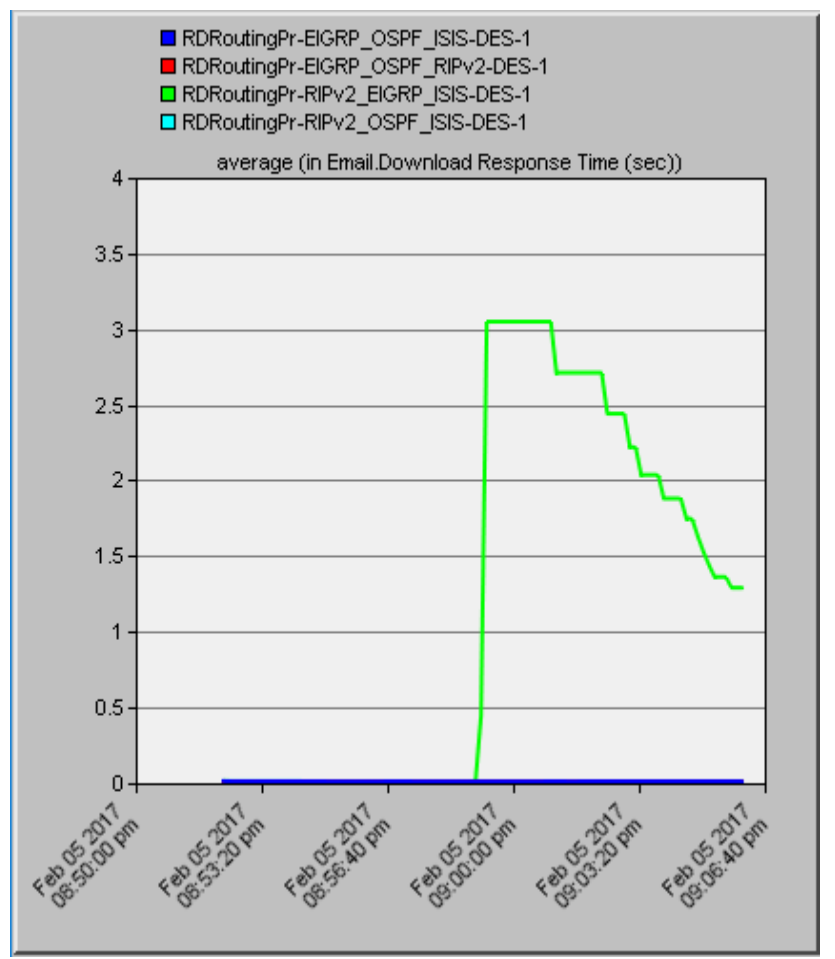

Fig. 12. The Email Download Response Time in the Four Scenarios 


\section{Voice Jitter}

The performance analysis in terms of multimedia service, the voice and video conferencing services are used in order to demonstrate the results in this study. Fig. 13 shows that the RIPv2_EIGRP_ISIS scenario is the best voice jitter from among the three other scenarios, where a jitter is a variation in delay time of received packets. In contrast, the EIGRP_OSPF_RIPv2 scenario is the worst one.

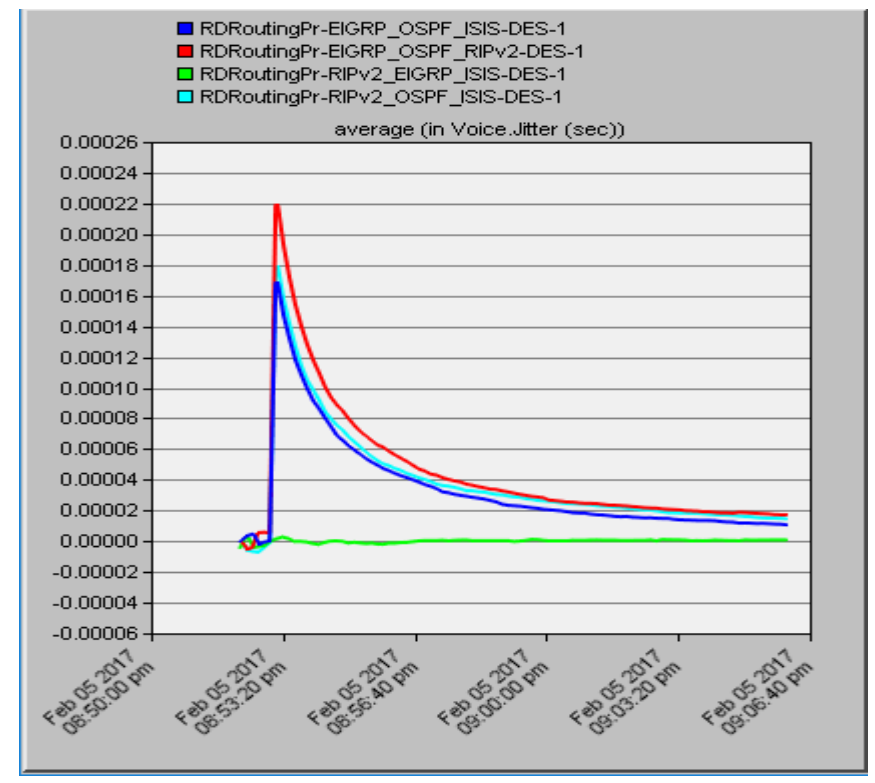

Fig. 13. The Voice Jitter in the Four Scenarios

\section{E. Voice Packet Delay Variation}

The RIPv2_EIGRP_ISIS scenario outperforms the three other scenarios in terms of the voice packet delay variation that has less delay variation as shown in Fig. 14, where a delay variation is a delay in receiving packets at the receiver. In the other hand, the EIGRP_OSPF_ISIS scenario has the worst result.

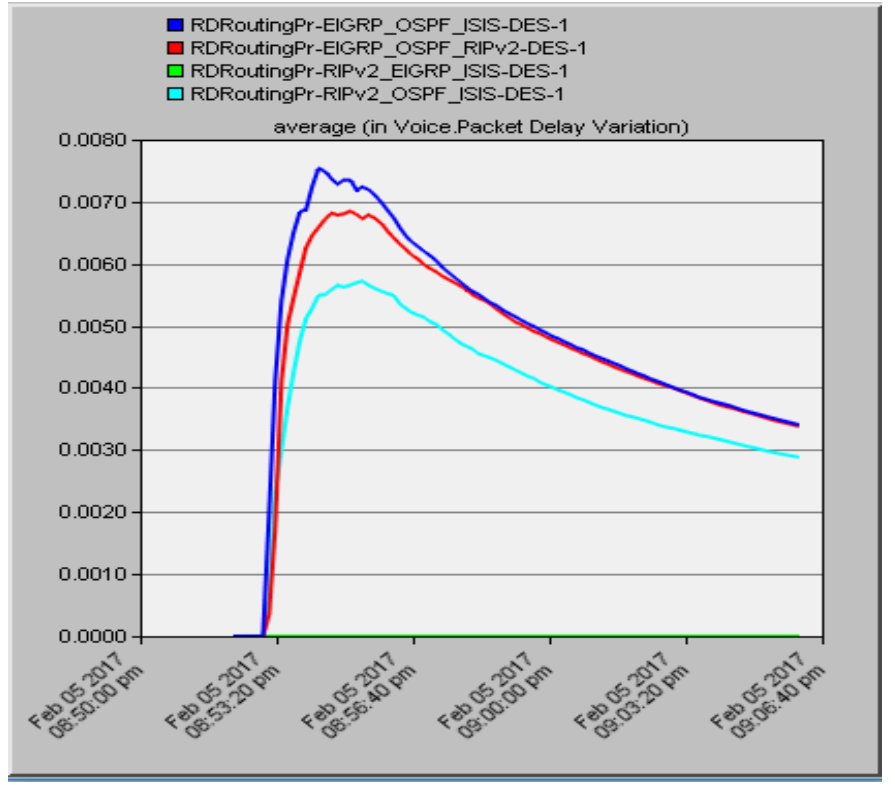

Fig. 14. The Voice Packet Delay Variation in the Four Scenarios

\section{F. Voice Packet End to End Delay}

The Fig. 15 shows the RIPv2_EIGRP_ISIS scenario that has less end to end delay time as compared with the three other scenarios, where end to end delay is defined as the time taken for a packet to be sent via a network from sender to receiver.

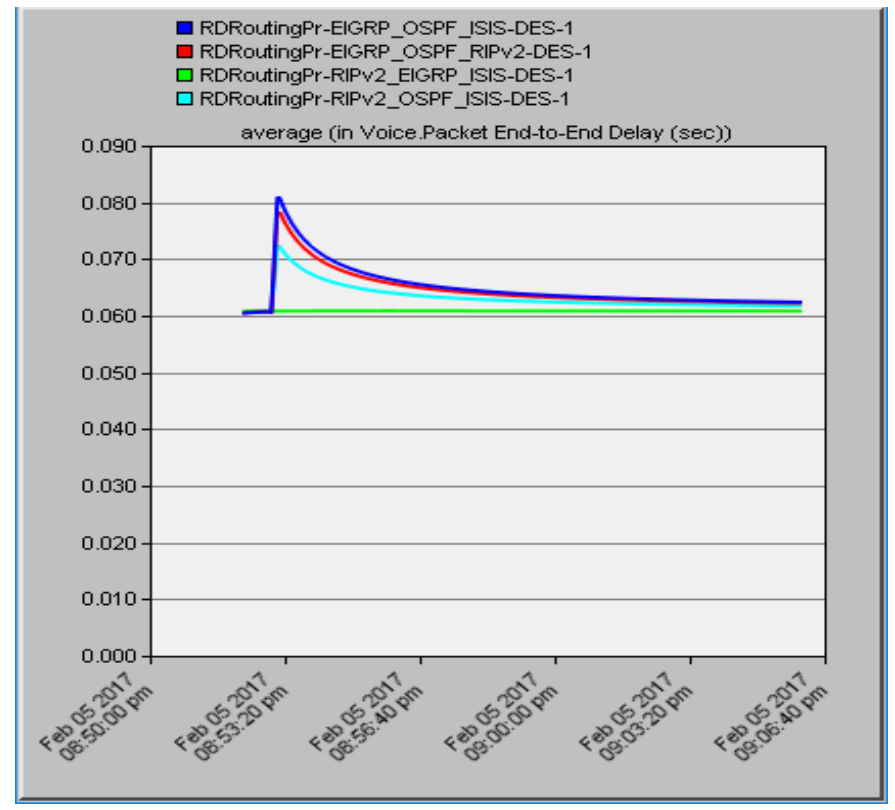

Fig. 15. The Voice Packet End to End Delay in the Four Scenarios

\section{G. Video Conferencing Packet Delay Variation}

The RIPv2_EIGRP_ISIS scenario has less delay variation as compared with the three other scenarios, therefore, it is the best one in terms of video conferencing service. On the other hand, the route redistribution among three protocols EIGRP, OSPF, and RIPv2 in the EIGRP_OSPF_RIPv2 scenario that has the worst result in the simulation as shown in Fig. 16.

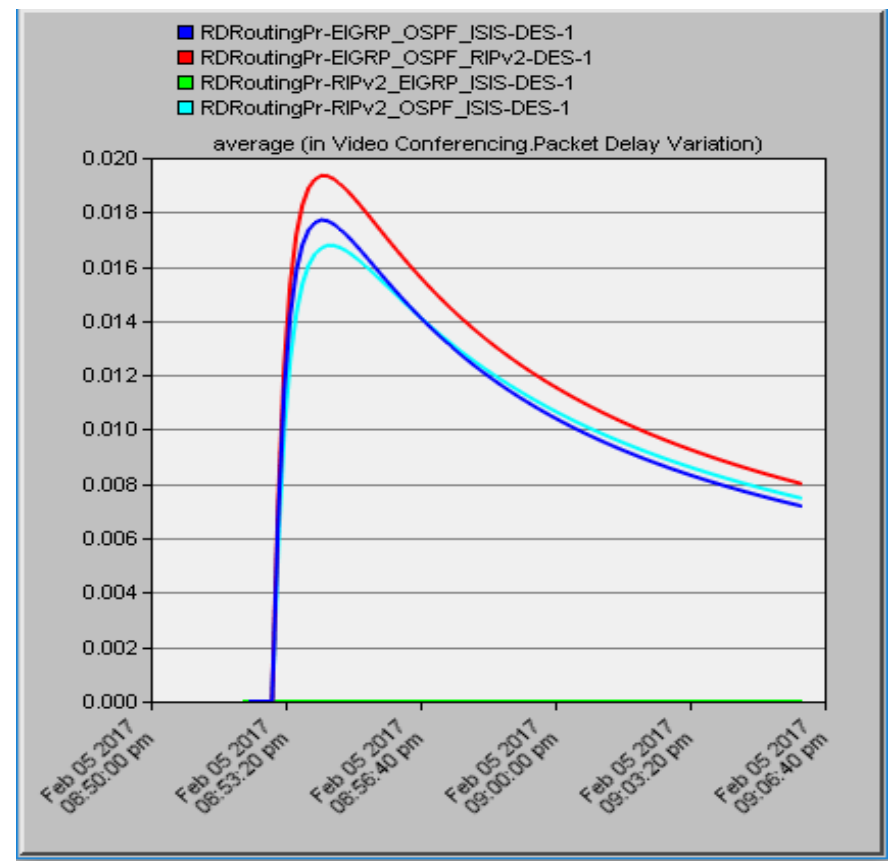

Fig. 16. The Video Conferencing Packet delay Variation in the Four Scenarios 


\section{H. Video Conferencing Packet End to End Delay}

The video conferencing packet end to end delay is demonstrated in Fig. 17. According to this figure, the RIPv2_EIGRP_ISIS scenario, before of failure occurrence and after network recovery that it has the lowest value and stable compared with the other scenarios consequently it is the best in terms of real time application.

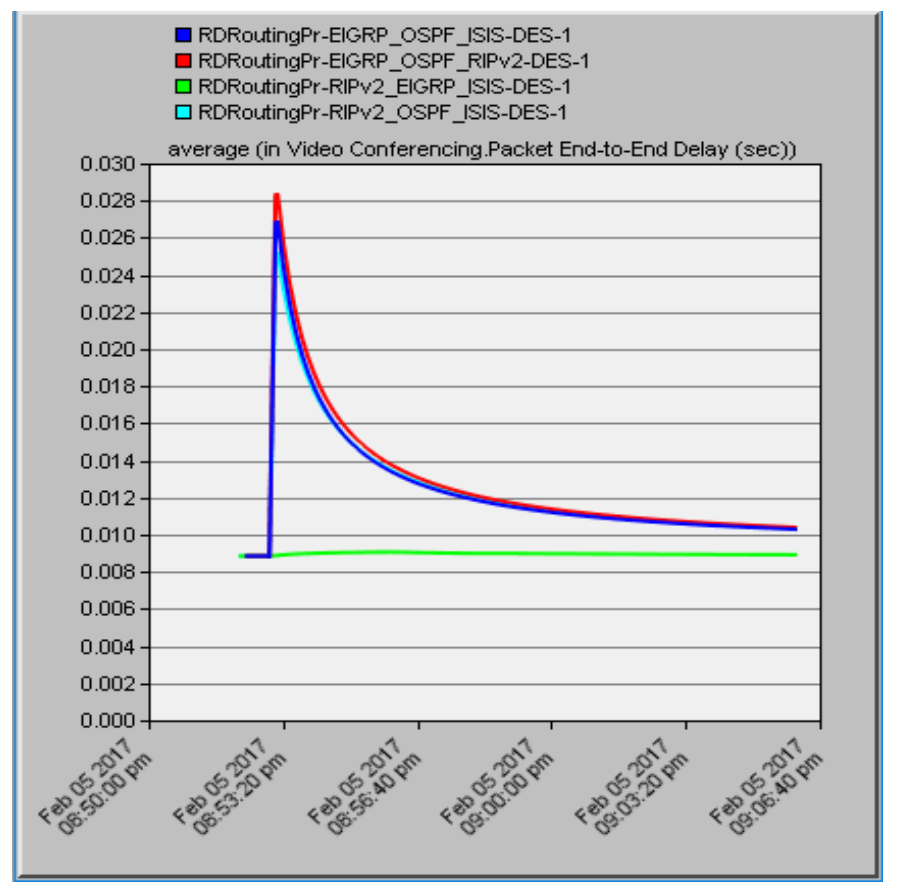

Fig. 17. The Video Conferencing Packet End to End Delay in the Four Scenarios

\section{CONCLUSION AND FUtURE WORK}

The Large network infrastructure consists of multiple routing protocols in order to advertise different routing information, therefore, the network border routers should be configured in order to keep the network connected. This paper has analyzed the performance of the route redistribution among three different routing protocols. The four scenarios are created and configured on the same network from different dynamic routing protocols. The first scenario is configured from EIGRP, OSPF, and IS-IS, the route redistribution among EIGRP, OSPF, and RIPv2 that is configured in the second scenario, the third scenario is the combination of RIPv2, EIGRP, and IS-IS, and the last scenario is configured from RIPv2, OSPF, and IS-IS routing protocols. The simulation showed the third scenario RIPv2_EIGRP_ISIS that is the best in terms of real time application such as voice and video conferencing as compared with the other scenarios in this study. The RIPv2_EIGRP_ISIS scenario has the optimal path of the hops number and minimal value of convergence duration time. In contrast, the EIGRP_OSPF_ISIS scenario has fast response time in terms of using Email, database query, and web browsing services.

In the future work, the route redistribution among three different dynamic routing protocols in this paper which will be tested on the GNS3 emulator.

\section{REFERENCES}

[1] F. Le, G. G. Xie and H. Zhang, "Understanding Route Redistribution," 2007 IEEE International Conference on Network Protocols, Beijing, 2007, pp. 81-92.

[2] Xin Sun, Sanjay G. Rao, and Geoffrey G. Xie. "Modeling complexity of enterprise routing design." In Proceedings of the 8th international conference on Emerging networking experiments and technologies, Nice, 2012, pp. 85-96.

[3] David A. Maltz, Geoffrey Xie, Jibin Zhan, Hui Zhang, Gísli Hjálmtýsson, and Albert Greenberg, "Routing design in operational networks: a look from the inside", In Proceedings of the 2004 conference on Applications, technologies, architectures, and protocols for computer communications, Portland, 2004, pp. 27-40.

[4] Cisco, Redistributing Routing Protocols, Mar, 2012.

[5] Cisco, OSPF Design Guide, Aug, 2005.

[6] Cisco, Intermediate System-to-Intermediate System Protocol.

[7] Mustafa Abdulkadhim, "Routing Protocols Convergence Activity and Protocols Related Traffic Simulation With It's Impact on the Network", IJCSET. International Journal of Computer Science Engineering and Technology, vol. 5, no. 3, pp. 40-43, March, 2015.

[8] Anibrika Bright Selorm Kodzo, Mustapha Adamu Mohammed, Ashigbi Franklin Degadzor and Dr. Michael Asante, "Routing Protocol (EIGRP) Over Open Shortest Path First (OSPF) Protocol with Opnet", IJACSA. International Journal of Advanced Computer Science and Applications, vol. 7, no. 5, pp.77-82, 2016

[9] Lalu Zazuli Azhar Mardedi and Abidarin Rosidi, "Developing Computer Network Based on EIGRP Performance Comparison and OSPF", IJACSA. International Journal of Advanced Computer Science and Applications, vol. 6, no. 9, pp. 80-86, 2015.

[10] Richard John Whitfield and Shao Ying Zhu,"A Comparison of OSPFv3 and EIGRPv6 in a Small IPv6 Enterprise Network", IJACSA. International Journal of Advanced Computer Science and Applications, vol. 6, no. 1, pp.162-167, 2015.

[11] G. K. Dey, M. M. Ahmed and K. T. Ahmmed, "Performance analysis and redistribution among RIPv2, EIGRP \& OSPF Routing Protocol", International Conference on Computer and Information Engineering, Rajshahi, 2015, pp. 21-24.

[12] Haresh N. Patel and Prof.Rashmi Pandey, "Enhanced Analysis on Route Summarization and Route Redistribution with OSPF vs. EIGRP Protocols Using GNS-3 Simulation", IJCTA. Int.J.Computer Technology \& Applications, vol. 5, no. 5, pp. 1682-1689, ,Sept-Oct, 2014.

[13] S. Farhangi, A. Rostami and S. Golmohammadi, "Performance Comparison of Mixed Protocols Based on EIGRP, IS-IS and OSPF for Real-time Applications", Middle-East Journal of Scientific Research, vol. 12 , no. 11, pp. 1502-1508, 2012.

[14] Syed Yasir Jalali, Sufyan Wani and Majid Derwesh, "Qualitative Analysis and Performance Evaluation of RIP, IGRP, OSPF and EGRP Using OPNETTM", Advance in Electronic and Electric Engineering, vol. 4, no. 4, pp. 389-396, 2014.

[15] Asmaa Shaker Ashoor, "Performance Analysis Between Distance Vector Algorithm (DVA) \& Link State Algorithm (LSA) For Routing Network", International Jiurnal of Scientific \& Technology Research, vol. 4, no. 02, pp. 101-105, February, 2015.

[16] Martin Kuradusenge and Damien Hanyuwimfura, "Operation and Comparative Performance Analysis of Enhanced Interior Routing Protocol (EIGRP) over IPv4 and IPv6 Networks", International Journal of Advanced Research in Computer Science and Software Engineering, vol. 6, no. 7, pp.174-182, July, 2016.

[17] Sukhkirandeep Kaur and Roohie Naaz Mir, "Performance Analysis of Interior Gateway Protocols", Advanced Research in Electrical and Electronic Engineering, vol. 1, no. 5, pp. 59-63, 2014.

[18] Kuwar Pratap Singh, P. K. Gupta and G. Singh, "Performance Evaluation of Enhanced Interior Gateway Routing Protocol in IPv6 Network", International Journal of Computer Applications ,vol. 70, No.5, pp. 42-47, May, 2013. 
[19] M. Pavani, M. Sri Lakshmi and Dr. S. Prem Kumar, "A Review on the Dynamic Routing Protocols in TCP/IP", The International Journal Of Science \& Technoledge, vol. 2, no. 5, pp.227-234, May, 2014

[20] P.Priyadhivya and S.Vanitha, "PERFORMANCE ANALYSIS OF INTERIOR GATEWAY PROTOCOLS", International Journal of Advanced Technology in Engineering and Science, vol. 3, No. 2, pp. 693-700 ,February, 2015.

[21] Shah.A and Waqas J.Rana, "Performance Analysis of RIP and OSPF in Network Using OPNET", IJCSI. International Journal of Computer Science Issues, vol. 10, Issue 6, No 2, pp. 256-265, November, 2013.

[22] S. Vissicchio, L. Vanbever, L. Cittadini, G. G. Xie and O. Bonaventure, "Safe routing reconfigurations with route redistribution", IEEE INFOCOM 2014 - IEEE Conference on Computer Communications, Toronto, 2014, pp. 199-207..

[23] IKram Ud Din, Saeed Mahfooz and Muhammad Adnan, "Analysis of the Routing Protocols in Real Time Transmission: A Comparative Study", Global Journal of Computer Science and Technology, vol. 10, no. 5, pp. 18-22, July, 2010.

[24] Jagmeet Kaur and Er. Prabhdeep Singh, "COMPARATIVE STUDY OF OSPFV3, IS-IS AND OSPFV3_IS-IS PROTOCOLS USING OPNET", IJARCET. International Journal of Advanced Research in Computer Engineering \& Technology, vol. 3, no. 8, pp. 2656-2662, August, 2014.
[25] ShewayeSirika and SmitaMahajine, "Performance Evaluation of Dynamic Routing Protocols for Real time application", IJETT. International Journal of Engineering Trends and Technology,vol. 32 no. 7, pp. 328-337 ,February, 2016.

[26] Komal Gehlot and N.C. Barwar, "Performance Evaluation of EIGRP and OSPF Routing Protocols in Real Time Applications", IJETTCS. International Journal of Emerging Trends \& Technology in Computer Science, vol. 3, no. 1, pp. 137-143, January - February, 2014.

[27] Y. N. Krishnan and G. Shobha, "Performance analysis of OSPF and EIGRP routing protocols for greener internetworking", ICGHPC. International Conference on Green High Performance Computing, Nagercoil, 2013, pp. 1-4..

[28] Moh'd Rasoul Ahmad Al-Hadidi, Mohammed Yousef Al-Gawagzeh, Nayel Al-Zubi,Bayan Al-Saaidah and Mohammed Alweshah, "Performance Analysis of EIGRP via OSPF Based on OPNET and GNS3", Research Journal of Applied Sciences, Engineering and Technology, vol. 8, no. 8, pp. 989-994, 2014.

[29] Jaswinder Kumar, Samiksha, Susil Bhagat and Karanjit Kaur," Route Redistribution between EIGRP and OSPF Routing Protocol in Computer Network Using GNS3", IJCNWMC. International Journal of Computer Networking, Wireless and Mobile Communications, vol. 5, no. 1, pp. 27-34, Feb, 2015. 